\title{
Investigation of Size Effects on the Al Nanoclusters Physical Properties via Molecular Dynamics Simulations
}

\author{
Mohammad Reza Sovizi, Ali Reza Madram \\ Department of Chemistry, Malek Ashtar University of Technology, Tehran, Iran \\ Email: mrsovizi@yahoo.com
}

Received 11 April 2014; revised 21 May 2014; accepted 30 May 2014

Copyright (c) 2014 by authors and OALib.

This work is licensed under the Creative Commons Attribution International License (CC BY). http://creativecommons.org/licenses/by/4.0/

c) (7) Open Access

\begin{abstract}
Molecular dynamics (MD) simulation has been carried out for study of size effects on physical properties of $\mathrm{Al}$ nanoclusters with different sizes $(\mathrm{N}=256,500,864,1372,2048$ and 4000) in the temperature range $300 \mathrm{~K} \leq \mathrm{T} \leq 1200 \mathrm{~K}$ for both free and periodic boundary conditions. Energy per site for periodic and free boundary conditions was calculated for the mentioned sizes and temperature. By increasing size of Al nanocluster, the energy per atom was convergence to that of the bulk. By use of molecular dynamics some physical properties such as melting point, surface energy, sublimation energy, thermodynamic limit and pair distribution function have been obtained. Melting point of bulk state is $875 \mathrm{~K}$ which proportional the jump of energy and sharp peak in heat capacity. Our calculated sublimation energy $\left(\Delta H_{\text {s-calculated }}=330 \mathrm{~kJ} / \mathrm{mol}\right)$ was in good agreement with experimental result $\left(\Delta H_{\text {s-experimental }}=318 \mathrm{~kJ} / \mathrm{mol}\right)$.
\end{abstract}

\section{Keywords}

Molecular Dynamics Simulation, Surface Energy, Al Nanocluster, Physical Properties

\section{Introduction}

Metal particles with nanometer size containing tens to thousands of atoms; have attracted a great deal of interests because of a number of their exciting potential applications in catalysis as well as in electronic and optical nanodevices [1]-[3]. With the decreasing of particle size to nano scale level, they are formed by a limited reduced number of atoms and a large fraction of them occupy its surfaces. The strong size effects resulted from this limitation reduction process may lead to novel and different behaviors. The size and shape are determined unique properties of metal particles [4]. 
There are many studies which have investigated structural and dynamical behavior of different materials. This method has been proven to be one of the most powerful tolls to predict and reproduce experimental result [5]-[7].

Knowledge of their shape and precise structure would be very useful in the determination of their stability and related properties. Nevertheless, there are few studies to address a thorough energetic and thermal analysis of a wide variety of nanoparticles.

In recent decades, consolidate solids composed of nanometer size clusters have been attended because they have unique properties such as electrical, magnetic and thermomechanical properties [8] [9]. In contrast to conventional polycrystalline solids, nanophase materials have a large fraction of atoms in the interfacial region, which have a dramatic effect on the structure and physical properties of these materials such as surface energy. The surface energy describe the energetic situation on the surface of solids and the interaction between the cohesive and adhesive forces which, in turn, indicate if wetting occurs [10] [11].

Molecular dynamics simulation for temperature-dependent behavior of solid copper has been studied by Alper and Politzer [12]. Isothermal isobaric molecular dynamics simulation of water has been done by Amrani et al. [13]. Dynamic coexistence melting is now well understood in terms of the minimum of the potential energy surface of nano particles [14]-[16]. Ferrando and Baletto [16] were reviewed structural properties of nanoclusters such as Energetic, thermodynamic and kinetic properties. Calvo et al. [17] were been studied of Al clusters melting in the size range of $49 \leq N \leq 62$ by computer simulation and compared with experimental results.

Melting of neutral and singly charged sodium clusters $\mathrm{Na}_{n}$ and $\mathrm{Na}_{n}{ }^{+}$was investigated for several sizes, $8 \leq \mathrm{n} \leq$ 139 using both Monte Carlo and molecular dynamics simulations with an empirical many-body potential by Calvo et al. [18]. Up to $n=75$, They found that the solid-liquid-like phase change is a multistage process initiated by "pre melting" The equilibrium structure is found to play an important role in the thermodynamics, in particular when such pre melting features are induced by diffusion processes at the surface. However, larger sizes $n \geq 93$ exhibit preferentially a single process similar to the bulk melting.

Influence of the many-body interactions on the thermodynamics and chaotic dynamics of small atomic clusters has been investigated by Calvo et al. With standard molecular dynamics simulations as well as parallel tempering Monte Carlo, They calculated the complete heat capacity curves and the variations of the largest Lyapunov exponent with total energy. They observed that increasing the magnitude or decreasing the range of the many-body forces leads to an increase of chaos and a decrease in the melting temperature. Decreasing the range of the many-body interactions also results in a significantly lower latent heat of melting [19]. Also molecular dynamics simulations were performed to determine the melting point of aluminum nanoparticles of 55 1000 atoms by the Streitz and Mintmire [20] [21].

In this letter, we will address the size effects on the thermal characteristics of Al nanoparticles by molecular dynamics simulations.

\section{Molecular Dynamics Simulation}

The MD simulations for Al bulk are carried out in a NVT ensemble with a constant number of atoms $N$, volume $V$, and temperature $T$ with the periodic boundary conditions, and for Al nanoclusters are carried out in a $N V E$ ensemble without any periodic boundary conditions. Temperature is controlled by a Nose-Hoover thermostat [22] and the equations of motion are integrated using the Verlet Leapfrog algorithm [23] with a time step of $0.001 \mathrm{ps}$. The system was equilibrated for $400 \mathrm{ps}(400,000$ time steps), the averages have been computed over the following 500 ps (500,000 time steps).

In the present study, we have used the DL-POLY-2.20 program [24]. For construction of initial configuration, first, a FCC block of $\mathrm{Al}$ was constructed from a FCC unit cell by replication in three dimensions with the center located at $(0,0,0)$. The fractional coordinates in the FCC structure are $(0,0,0),(0.5,0.5,0),(0.5,0,0.5)$, and $(0$, $0.5,0.5)$.

In the molecular dynamics simulations, we chose Sutton-Chen (SC) type potentials to describe interatomic interactions among metal atoms in a nanoparticle. This potential represents the many-body interactions, and their parameters are optimized to describe the lattice parameter, cohesive energy, bulk modulus, elastic constants, phonon dispersion, vacancy formation energy, and surface energy, leading to an accurate description of many properties of metals and their alloys [25]-[28]. For the SC-type force field, the total potential energy for a system of atoms can be written as 


$$
\sum_{i=}^{N} U_{i}=\sum_{i}^{N} \varepsilon\left[1 / 2 \sum_{j}^{N} V\left(R_{i j}\right)-c \sqrt{\rho_{i}}\right]
$$

Here $V\left(R_{i j}\right)$ is a pair interaction function defined by the following equation

$$
V\left(R_{i j}\right)=\left(a / R_{i j}\right)^{n}
$$

Accounting for the repulsion between the $i$ and $j$ atomic cores; $\rho_{i}$ is a local electron density accounting for cohesion associated with atom $i$ defined by

$$
\rho=\sum_{j \neq i}\left(\frac{a}{r_{i j}}\right)^{m}
$$

In Equations (1) and (2), $R_{i j}$ is the distance between atoms $i$ and $j, a$ is a length parameter scaling all spacing (leading to dimensionless $V$ and $\rho$ ); $c$ is a dimensionless parameter scaling the attractive terms; $\varepsilon$ sets the overall energy scale; $n$ and $m$ are integer parameters such that $n>m$. Given the exponents $(n, m), c$ is determined by the equilibrium lattice parameter, and $\varepsilon$ is determined by the total cohesive energy. The values of $\varepsilon, a, n, m, c$ for $\mathrm{Al}$ are: $0.033147(\mathrm{eV}), 4.05\left(\mathrm{~A}^{0}\right), 7.0,6.0,16.399$, respectively [23].

\section{Result and Discussion}

\subsection{Thermodynamics Limit}

Molecular dynamics simulation has been carried out for $\mathrm{Al}$ nanoclusters with different sizes $(N=256,500,864$, 1372, 2048 and 4000) in the temperature range $300 \mathrm{~K} \leq \mathrm{T} \leq 1200 \mathrm{~K}$ for both free and periodic boundary conditions. Energy per site for periodic and free boundary conditions is calculated for the mentioned sizes and temperature. By increasing size of Al nanoclusters, the energy per atom convergence to that of the bulk. To obtain thermodynamic limit, we have calculated the equilibrium configurationally energy per site for the bulk at $300 \mathrm{~K}$. The percent difference as a function of cluster size is shown in Figure 1.

On the basis of Figure 1, by increasing the cluster size, the magnitude for the difference in configurationally energy per site of the bulk and nanocluster decreases with $N$ and approaches to zero exponentially. The percent difference is about 3.5 for $N=4000$.

\subsection{Surface Energy}

The reversible work per unit area to form a new surface of a substance (for instance by cleavage) is defined as the specific surface free energy (or simply called the surface free energy $\gamma$ ), while the reversible work per unit area required toelastically stretch a surface is the surface tension, $\sigma$. The surface free energy constitutes a noticeable contribution to the total free energy of a nanoparticle.

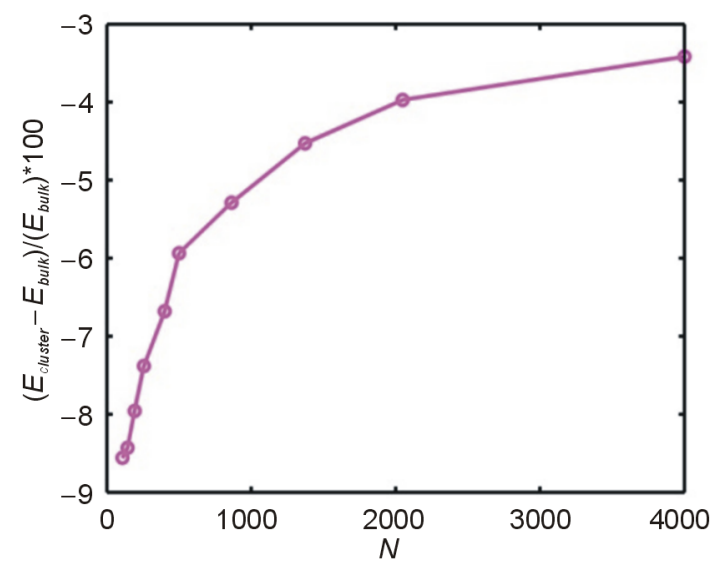

Figure 1. Percent difference in energy per site of the nanoclusters, compared to the bulk value, as a function of cluster size. 
For solids, usually the surface tension is not equal to the surface free energy in value. Thus $\gamma$ for a solid may be computed as

$$
\sigma=\gamma+A_{s} \frac{\partial \gamma}{\partial A_{s}}
$$

where $\sigma$ is the surface tension, represents the surface free energy and $A_{s}$ is the surface area. To calculate the surface free energy, we use the approach given in reference [29].

The surface energy of a cluster may be calculated from

$$
\gamma=\frac{E_{\text {cluster }}-E_{\text {bulk }}}{4 \pi R_{c}^{2}}
$$

where $E_{\text {cluster }}$ and $E_{\text {bulk }}$ are the potential energies per site of cluster and bulk, respectively, and $R_{c}$ is the cluster radius at a certain temperature. We have calculated the surface energy (per unit surface area) and cluster radiuses, at $300 \mathrm{~K}$ for different sizes of nanocluster 108 - 4000, the results are shown in Figure 2.

According to the Figure 2, the surface energy exponentially decreases with the cluster size.

\subsubsection{Melting Point of Nanocluster}

The configurationally energy per size for the $\mathrm{Al}$ nanocluster with different sizes 108, 144, 192, 256, 320, 400, 500, 864, 1372, 2048 and 4000 is calculated at different temperatures $300 \mathrm{~K} \leq \mathrm{T} \leq 1200 \mathrm{~K}$. To identify the melting temperature, we have calculated the specific heat capacity at constant volume $\mathrm{C}$ as,

$$
C_{v}=\left(\frac{\partial E}{\partial T}\right)_{v}
$$

A jump in the calculated heat capacity as a function of temperature indicates the melting point. The temperature range in which the melting occurs for different cluster is summarized in Table 1.

Our results for the melting points of $\mathrm{Al}$ nanocluster shows good agreements these given in reference [21].

We have taken 3 snapshots for an Al nanocluster with $N=256$ at three different temperatures $20 \mathrm{~K}, 500 \mathrm{~K}$, and $800 \mathrm{~K}$

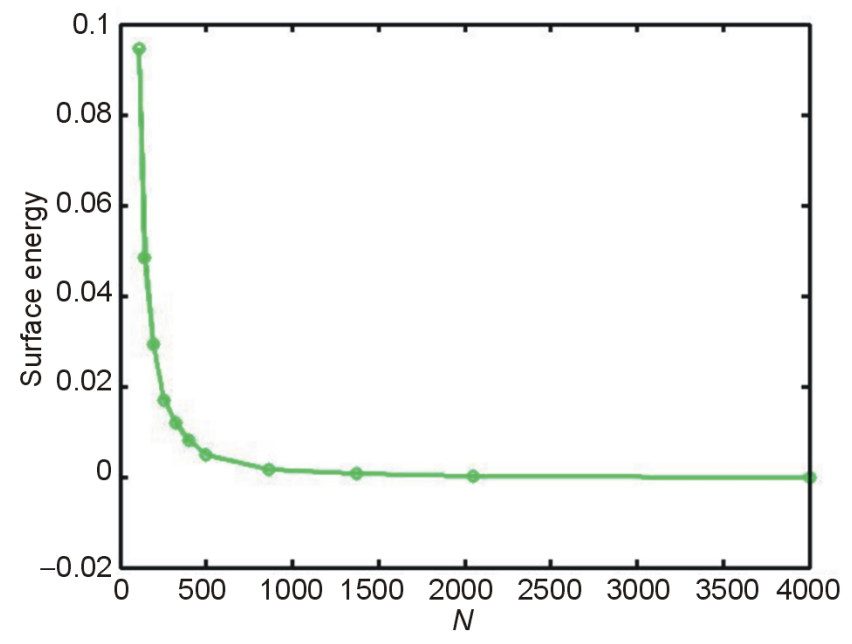

Figure 2. Dependence of the surface energy (eV/NA2) to the cluster size.

Table 1. The range of melting point temperature for the given $\mathrm{Al}$ nanoclusters.

\begin{tabular}{cccccccccccc}
$\begin{array}{c}\text { Size } \\
\text { (Atom) }\end{array}$ & 108 & 144 & 192 & 256 & 300 & 400 & 500 & 864 & 1372 & 2048 & 4000 \\
\hline $\begin{array}{c}\text { Melting } \\
\text { Point (K) }\end{array}$ & $420-470$ & $520-560$ & $530-560$ & $540-570$ & $560-590$ & $570-590$ & $580-600$ & $600-620$ & $640-650$ & $870-872$ & $874-875$ \\
\hline
\end{tabular}


According to Figure 3 the $\mathrm{Al}$ nanocluster with 256 atoms at the lowest temperature $20 \mathrm{~K}$ has an ordered phase, when temperature increases at $500 \mathrm{~K}$ before melting point, the solid of nanocluster becomes disordered, and finally at $800 \mathrm{~K}$ above the melting point the structure of nanocluster is destroyed.

The correlation radial distribution function $g(r)$ is calculated at the three temperatures shown in Figure 4.

On the basis of Figure 4 at the lowest temperature the nanocluster has a very high peak, even at large distance there are some smaller peaks which indicates the long range order in nanocluster. By increasing temperature the high peak becomes smaller.

\subsubsection{Melting Point of Bulk}

Configurationally energy per size of Al bulk using 2048 atom and periodic boundary condition is calculated at different temperatures within the $300 \leq \mathrm{T} \leq 1200$. The calculated configurationally energy is plotted versus temperature in Figure 5. On the basis of Figure 5, there is a jump in the configuration energy thoroughly at 875 $\mathrm{K}$.

The calculated heat capacity of the bulk is plotted versus temperature in Figure 6 for 2048 atoms $\mathrm{Al}$ in bulk. The maximum which appears in the specific heat capacity corresponds to the melting point of the bulk.

\subsection{Sublimation Energy}

Molecular Dynamics simulations have been done at zero pressure; therefore internal energy is equal to enthalpy. Namely:

$$
H=U+P V=U
$$

We may assume that $\mathrm{Al}$ in gas phase is ideal and then sublimation energy may be given by

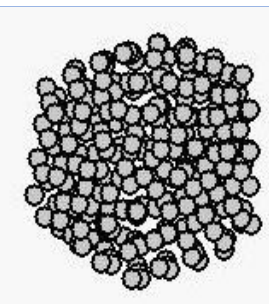

(a)

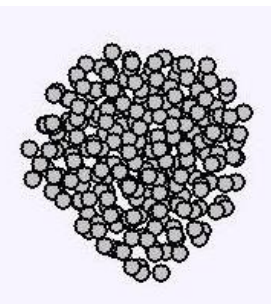

(b)

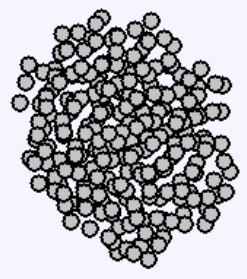

(c)

Figure 3. Snapshot of an $\mathrm{Al}$ nanocluster with $\mathrm{N}=256$ at three different temperatures: at (a) 20, (b) 500 and (c) $800 \mathrm{~K}$.

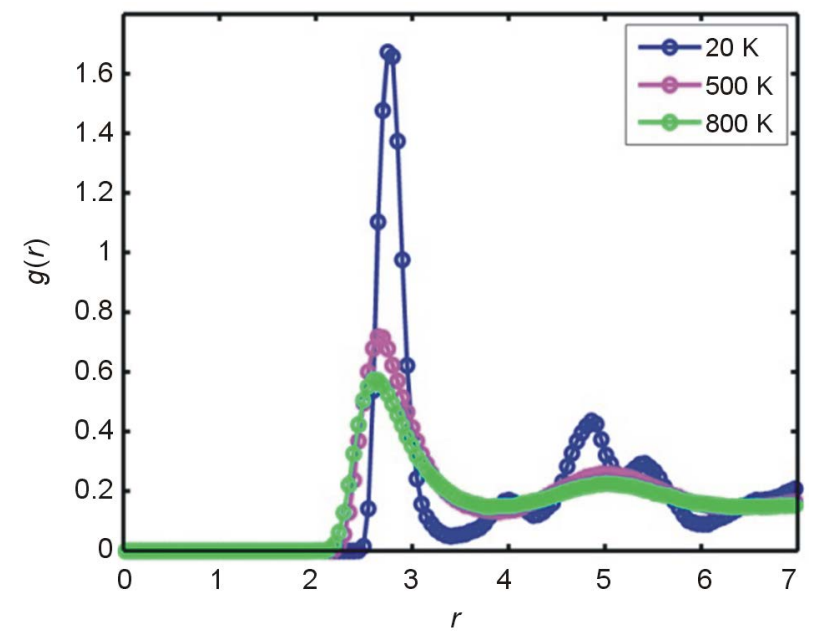

Figure 4. Pair correlation function versus distance for the Alnanocluster with $\mathrm{N}=256$ at three different temperatures 20, 500 and $800 \mathrm{~K}$. 


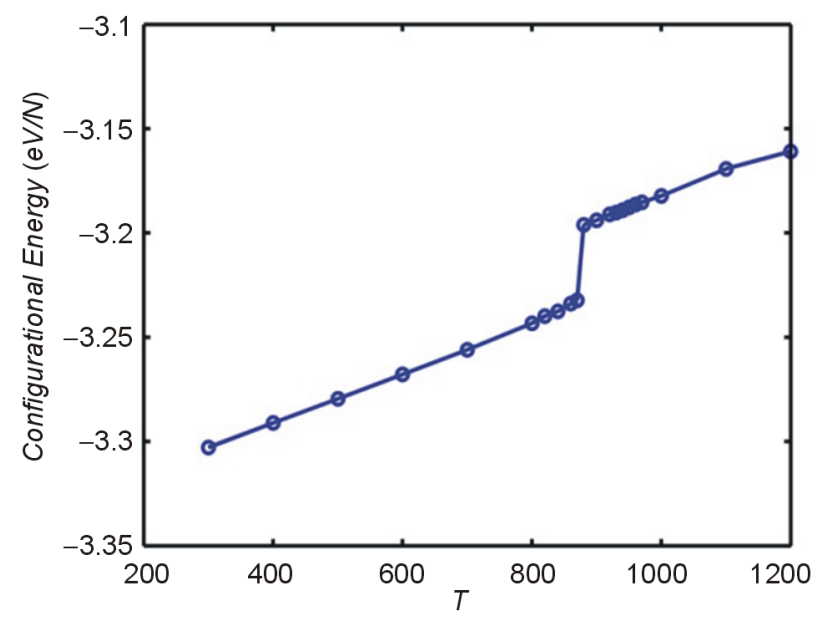

Figure 5. Configurationally energy per site for Al bulk versus temperature.

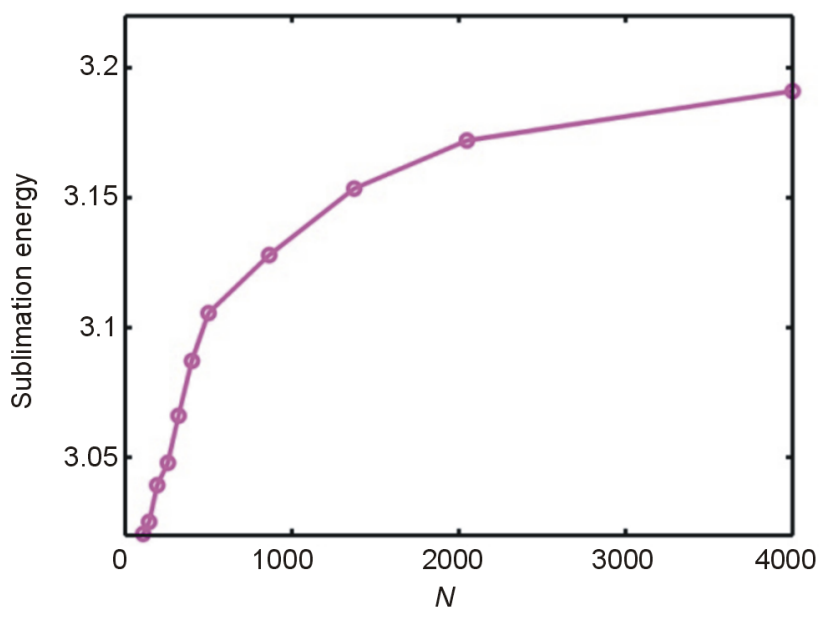

Figure 6. Heat capacity per size versus temperature for Al bulk.

$$
\Delta H_{s}=H_{g}-H_{s}=\frac{3}{2} N k_{B} T-U_{s}
$$

Equation (7) is used to calculate for the clusters with different size. The calculated sublimation energy is shown in Figure 7.

According to Figure 7 the sublimation energy increase with $N$ and asymptotically approach to limit of sublimation energy per size is $3.3 \mathrm{eV} / \mathrm{N}(318 \mathrm{~kJ} / \mathrm{mol})$. Experimental value for the sublimation energy was $330 \mathrm{~kJ} / \mathrm{mol}$ and it was closed to our result.

\section{Conclusion}

Molecular dynamics have been used for investigation of thermodynamic properties of $\mathrm{Al}$ in nanocluster and bulk states. Some physical properties such as melting point, surface energy, sublimation energy, thermodynamic limit and pair distribution function have been obtained. By increasing the size of nanocluster, configuration of energy approaches to thermodynamic limit. For instance, the relative error from the bulk state for a typical particle consisting $4000 \mathrm{Al}$ atom is roughly 3 percent. Surface free energy decreases exponentially as a function of size and its values is roughly zero at size of 1372 . The main peak of pair distribution function at low temperature (before melting) is sharp. There are also some other peaks after the main peak which may indicate existence of ordering in long distance. However, increasing the temperature will result in disappearing and broadening of all the 


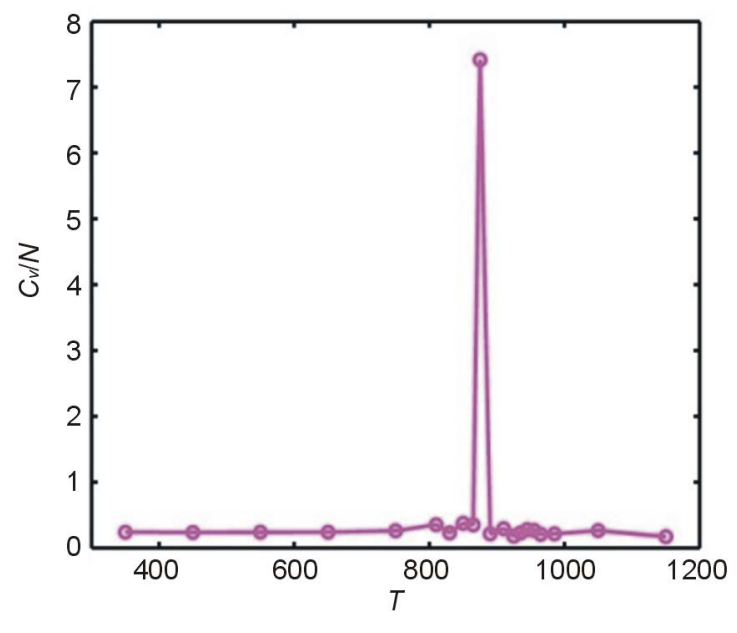

Figure 7. Sublimation energy versus size for the $\mathrm{Al}$ nanocluster.

peaks. Melting point of bulk state is $875 \mathrm{~K}$ which proportional the jump of energy and sharp peak in heat capacity. Our calculated sublimation energy is in good agreement with experimental result $\left(\Delta H_{s-\text { calculated }}=330 \mathrm{~kJ} / \mathrm{mol}\right.$, $\Delta H_{\text {s-experimental }}=318 \mathrm{~kJ} / \mathrm{mol}$ ).

\section{References}

[1] Zhdanov, V.P., Schwind, M., Zorić, I. and Kasemo, B. (2010) Overheating and Undercooling during Melting and Crystallization of Metal Nanoparticles. Physica E, 42, 1990-1994. http://dx.doi.org/10.1016/j.physe.2010.03.014

[2] Jensen, P. (1990) Growth of Nanostructures by Cluster Deposition: Experiments and Simple Models. Reviews of Modern Physics, 71, 1695-1735. http://dx.doi.org/10.1103/RevModPhys.71.1695

[3] Umeno, A. and Hirakawa, K. (2010) Spectroscopic Analysis of Electromigration at Gold Nanojunctions. Physica E, 42, 2826-2829. http://dx.doi.org/10.1016/j.physe.2009.12.057

[4] Zhdanov, V.P. and Kasemo, B. (2010) Apparent Kinetics of Hydriding and Dehydriding of Metal Nanoparticles. Physica E, 42, 1482-1486. http://dx.doi.org/10.1016/j.physe.2009.12.002

[5] Rouhi, S. and Ansari, R. (2012) Atomistic Finite Element Model for Axial Buckling and Vibration Analysis of SingleLayered Graphene Sheets. Physica E, 44, 764-772. http://dx.doi.org/10.1016/j.physe.2011.11.020

[6] Seyyed Fakhrabadi, M.M., Samadzadeh, M., Rastgoo, A., Haeri Yazdi, M. and Mousavi Mashhadi, M. (2012) Vibrational Analysis of Carbon Nanotubes Using Molecular Mechanics and Artificial Neural Network. Physica E, 44, 565578. http://dx.doi.org/10.1016/j.physe.2011.10.004

[7] Feng, C. and Jiang, L.Y. (2011) Molecular Dynamics Simulation of Squeeze-Film Damping Effect on Nano Resonators in the Free Molecular Regime. Physica E, 43, 1605-1609. http://dx.doi.org/10.1016/j.physe.2011.05.004

[8] Gleiter, H. (1992) Materials with Ultrafine Microstructures: Retrospective and Perspective. Nanostructured Materials, 1, 1-19. http://dx.doi.org/10.1016/0965-9773(92)90045-Y

[9] Kamenev, B.V., Baribeau, J.-M., Lockwood, D.J. and Tsybeskov, L. (2005) Optical Properties of Stranski-Krastanov grown Three-Dimensional Si/Si-xGex Nanostructures. Physica E, 26, 174-179. http://dx.doi.org/10.1016/j.physe.2004.08.047

[10] Kalia, R. K., Nakano, A., Tsuruta, K. and Vashishta, P. (1997) Morphology of Pores and Interfaces and Mechanical Behavior of Nanocluster-Assembled Silicon Nitride Ceramic. Physical Review Letters, 78, 689-692. http://dx.doi.org/10.1103/PhysRevLett.78.689

[11] Campbell, T., Kalia, R.K., Nakano, A. and Vashishta, P. (1999) Dynamics of Oxidation of Aluminum Nanoclusters using Variable Charge Molecular-Dynamics Simulations on Parallel Computers. Physical Review Letters, 82, 48664869. http://dx.doi.org/10.1103/PhysRevLett.82.4866

[12] Alper, H.E. and Politzer, P. (1999) Molecular Dynamics Simulation of the Temperature-Dependent Behavior of Solid Copper. Journal of Molecular Structure: THEOCHEM, 487,117-125. http://dx.doi.org/10.1016/S0166-1280(99)00147-5

[13] Amrani, M., Bendeddouch, D., Bormann, D. and Krallafa, A. (2008) Isothermal Isobaric Molecular Dynamics Simula- 
tion of Water. Journal of Molecular Structure: THEOCHEM, 867, 39-46. http://dx.doi.org/10.1016/j.theochem.2008.07.017

[14] Wales, D.J. and Berry, R.S. (1994) Coexistence in Finite Systems. Physical Review Letters, 73, 2875-2878. http://dx.doi.org/10.1103/PhysRevLett.73.2875

[15] Erkoç, Ş. (2000) Stability of Gold Clusters: Molecular-Dynamics Simulations. Physica E, 8, 210-218. http://dx.doi.org/10.1016/S1386-9477(00)00158-2

[16] Ferrando, R. and Baletto, F. (2005) Structural Properties of Nanoclusters: Energetic, Thermodynamic, and Kinetic Effects. Reviews of Modern Physics,77, 371-423. http://dx.doi.org/10.1103/RevModPhys.77.371

[17] Calvo, F., Noya, E.G., and Doye, J.P.K. (2006) Theoretical Study of the Melting of Aluminum Clusters. Physical Review B, 73, 125407-125413. http://dx.doi.org/10.1103/PhysRevB.73.125407

[18] Calvo, F. and Spiegelmann, F. (1999) Geometric Size Effects in the Melting of Sodium Clusters. Physical Review Letters, 82, 2270-2273. http://dx.doi.org/10.1103/PhysRevLett.82.2270

[19] Calvo, F. and Yurtsever, E. (2000) Many-Body Effects on the Melting and Dynamics of Small Clusters. Physical Review B, 62, 9977-9980. http://dx.doi.org/10.1103/PhysRevB.62.9977

[20] Streitz, F.H. and Mintmire, J.W. (1994) Electrostatic Potentials for Metal-Oxide Surfaces and Interfaces. Physical Review B, 50, 11996-12003. http://dx.doi.org/10.1103/PhysRevB.50.11996

[21] Alavi, S., Mintmire, J.W. and Thompson, D.L. (2005) Molecular Dynamics Simulations of the Oxidation of Aluminum Nanoparticles. Journal of Physical Chemistry B, 109, 1518-1523. http://dx.doi.org/10.1021/jp046196x

[22] Nose,S. (1990) Constant-Temperature Molecular Dynamics. Journal of Physics: Condensed Matter, 2, SA115-SA119. http://dx.doi.org/10.1088/0953-8984/2/S/013

[23] Allen, M.P. and Tildesley, D.J. (1997) Computer Simulation of Liquid. Oxford University, New York,

[24] Smith, W. and Todorov, I.T. (2006) A short description of DL_POLY. Molecular Simulation, 32, 935-943. http://dx.doi.org/10.1080/08927020600939830

[25] Qi, Y. Cagin, T., Johnson, W.L. and Goddard, W.A. (2001) Melting and Crystallization in Ni Nano-Clusters: The mesoscale regime. Journal of Chemical Physics, 115, 385-394. http://dx.doi.org/10.1063/1.1373664

[26] Wen, Y.H., Wu, S.Q., Zhang, J.H. and Zhu, Z.Z. (2008) The Elastic Behavior in Ni Monocrystal: Nonlinear Effects. Solid State Communications, 146, 253-257. http://dx.doi.org/10.1016/j.ssc.2008.02.020

[27] Sankaranarayanan, S.K.R.S., Bhethanabotla, V.R. and Joseph, B. (2005) Molecular Dynamics Simulation Study of the Melting of Pd-Pt Nanoclusters. Physical Review B, 71, 195415-195425. http://dx.doi.org/10.1103/PhysRevB.71.195415

[28] Vanfleet, R.R. and Mochel, J.M. (19950 Thermodynamics of Melting and Freezing in Small Particles. Surface Science, 341, 40-50. http://dx.doi.org/10.1016/0039-6028(95)00728-8

[29] Wang, L., Zhang, Y., Bian, X. and Chen, Y. (2003) Melting of Cu Nanoclusters by Molecular Dynamics Simulation. Physics Letters A, 310, 197-202. http://dx.doi.org/10.1016/S0375-9601(03)00263-9 